\title{
Down-staging ( $<$ pT2) of urothelial cancer at cystectomy after the diagnosis of detrusor muscle invasion (pT2) at diagnostic transurethral resection (TUR): is prediction possible?
}

\author{
Willemien Beukers • Titia Meijer • Cornelis J. Vissers • \\ Joost L. Boormans • Ellen C. Zwarthoff • \\ Geert J. L. H. van Leenders
}

Received: 16 April 2012 /Revised: 30 May 2012 / Accepted: 25 June 2012 /Published online: 10 July 2012

(C) The Author(s) 2012. This article is published with open access at Springerlink.com

\begin{abstract}
Urothelial cell carcinoma (UCC) with musculus detrusor (MD) invasion is treated by cystectomy. Subsequent pathologic evaluation of cystectomies does not reveal MD invasion $(<\mathrm{pT} 2)$ in a subgroup of patients. Our objective was to identify features at diagnostic transurethral resection (TUR) predicting down-staging $(<\mathrm{pT} 2)$ at cystectomy. Patients with pathologically confirmed MD invasion at TUR followed by cystectomy for UCC without (neo-) adjuvant therapy were included $(N=106)$. Slides of both TUR and cystectomy specimens were reviewed, and survival analyses were performed. In total, 27/106 (26\%) tumors were down-staged at cystectomy, of which $13(12 \%)$ had no residual tumor (pT0). There was no significant difference in age, gender, time interval between TUR and operation, number of slides sampled, and presence of TUR scar between down-staged $(<\mathrm{pT} 2)$ and pT2 UCC. At review of TUR specimens $(N=52)$ with UCC initially diagnosed as pT2, MD invasion was not confirmed in eight cases (15\%). One case showed extensive histiocytic reaction misinterpreted as UCC; in four cases, muscularis mucosae had been considered $\mathrm{MD}$, and in three cases, desmoplastic reaction mimicked MD. No histologic parameter at TUR was significantly associated with down-staging at cystectomy. Overall and disease-specific survival was not statistically different in down-staged and pT2 UCC. In conclusion, down-staging of
\end{abstract}

W. Beukers $\cdot$ T. Meijer $\cdot$ C. J. Vissers $\cdot$ E. C. Zwarthoff $\cdot$

G. J. L. H. van Leenders $(\triangle)$

Department of Pathology, Josephine Nefkens Institute,

Erasmus MC,

PO Box 2040, 3000 CA Rotterdam, The Netherlands

e-mail: g.vanleenders@erasmusmc.nl

J. L. Boormans

Department of Urology, Erasmus MC,

Rotterdam, The Netherlands
UCC $(<$ pT2) at cystectomy occurred in $26 \%$. At review of diagnostic TURs, MD invasion was not confirmed in $15 \%$. No clinical or pathologic parameter was predictive for down-staging at cystectomy. There was no difference in survival between down-staged and pT2-staged UCC.

Keywords Cystectomy · Urothelial cell carcinoma . Down-staging $\cdot$ pT0

\section{Introduction}

Bladder cancer is the fourth most common cancer in males and ninth most common cancer in females [1]. The majority of bladder tumors primarily appear as non-muscle invasive disease $(<\mathrm{pT} 2)$, whereas $20 \%$ of urothelial carcinomas (UCCs) are infiltrating the musculus detrusor (MD) at presentation. Up to $25 \%$ of initially non-muscle invasive tumors will eventually progress to MD invasive disease. Tumors limited to the mucosa $(\mathrm{pTa} / \mathrm{pT} 1)$ are conservatively treated, while bladder cancer infiltrating within or beyond the MD ( $\geq \mathrm{pT} 2)$ requires aggressive treatment $[2,3]$. After radical surgery, the 5-year recurrence free-survival of $\geq \mathrm{pT} 2$ UCC is only $56-58 \%[4,5]$. Accurate pathologic staging of UCC at diagnostic transurethral resection (TUR) specimens is, therefore, an important parameter to determine the optimal therapy for individual patients.

Although accurate pathological staging at diagnostic TUR is pivotal for therapeutic decision-making, definite tumor stage can only be perceived after pathologic evaluation of cystectomy specimens. In a substantial number of patients, no invasion in the MD is identified at cystectomy, prompted by MD invasion at diagnostic TUR. These patients either show residual UCC without stromal invasion 
(pTa), UCC with invasion of the lamina propria (pT1), carcinoma in situ (CIS) (pTis), or no residual tumor at all (pT0). Down-staging of UCC to pT0 in cystectomy specimens is reported in 5 to $20 \%$ [6-10]. Although downstaging is a well-known feature, it can be confronting for patient, urologist, and pathologist, raising the question whether the initial diagnosis prompting radical surgery was adequate.

Several pathologic features might mimic MD invasion of UCC. Infiltration of the muscularis mucosae (MM) and extensive desmoplastic stromal reaction can both be misinterpreted as MD invasion. Furthermore, it is not wellestablished whether UCC located directly adjacent to but not within the MD should be staged as pT2 disease. The objective of the present study was (a) to identify clinical and pathological features of UCC at diagnostic TUR, which correspond with down-staging at cystectomy and (b) to investigate the clinical outcome of patients with downstaged UCC after cystectomy.

\section{Material and methods}

\section{Study population}

In this study, we included patients with pathologically confirmed MD invasion at diagnostic TUR, followed by cystectomy for UCC without neo-adjuvant chemotherapy or radiotherapy. All patients were treated for UCC of the urinary bladder at Erasmus MC, location Dijkzigt, between June 1989 and May 2010. Patients were routinely followed up every 3 months on the first 2 years after cystectomy, semiannually on the third to fifth year, and subsequently at 12-month intervals. In case of progression, patients were followed up every 3 months. Loco-regional recurrent disease or distant metastases were assessed by CT of the abdomen and pelvis, chest X-rays, and bone scans (if indicated). Death was reported by treating physicians or general practitioner. Follow-up was defined as time from operation to event or last patient contact. Patients that deceased from other causes than bladder cancer, or that were lost to followup, were censored at the date of last contact. All clinical data were collected retrospectively from the electronic patient database.

\section{Pathologic review}

Slides of cystectomies and diagnostic TUR specimens were retrieved from the archives and reviewed by a urogenital pathologist $(\mathrm{GvL})$. Initial diagnoses had been made by a group of general clinical pathologists. At review, stage and grade were reassigned according the UICC 2002 TNM staging system and the WHO grading system 1973 and
2004, respectively. The following additional pathological parameters were recorded at review of the cystectomy specimens: number of slides of the urinary bladder sampled, presence of a TUR scar, and presence of CIS. At review of the diagnostic TUR specimens, the depth of invasion was established paying special attention to potential pitfalls such as MM invasion, extensive desmoplastic stromal reaction, invasion adjacent to but not within the MD, and invasion in between MD bundles. If classification was uncertain based on routine haematoxylin and eosin staining (HE), immunohistochemistry was performed. In case MD invasion was not obvious despite histology and additional immunohistochemistry, the tumor was classified as pT1 $(N=1)$, which is similar to our approach in daily practice, in which these tumors are reported as "pT1 at least." The use of the samples for research purposes was approved by the Erasmus MC Medical Ethics Committee according to the Medical Research Involving Human Subjects Act (MEC-2004-261).

\section{Immunohistochemistry}

Formalin-fixed, paraffin-embedded tissue sections $(5 \mu \mathrm{m})$ from TUR specimens were cut and mounted on aminoacetylsilane-coated slides (Starfrost, Berlin, Germany). Sections were deparaffinised in EZprep (Roche Diagnostics, Indianapolis, IN, USA). For all antibodies, heat-induced epitope retrieval was performed in $\mathrm{CC} 1$ buffer (high $\mathrm{pH}$ ) (Roche Diagnostics) for $64 \mathrm{~min}$. The slides were incubated with monoclonal Desmin antibody (clone DE-R-11; 1:50; Novocastra, Newcastle, UK) or monoclonal keratin 7 antibody (clone OV-TL 12/30; 1:1,000; Biogenex, San Ramon, CA, USA) at $36{ }^{\circ} \mathrm{C}$ for $32 \mathrm{~min}$, followed by amplification using UltraView Universal DAB detection kit (Roche Diagnostics). All reactions were performed in an automatic BenchMark Ultra device (Roche Diagnostics). Finally, the slides were counterstained, dehydrated, cleared in xylene, and mounted in Pertex (Histolab Products, Gothenburg, Sweden).

\section{Statistical analysis}

For comparison of continuous and categorical parameters of the pT2 and the down-staged group at cystectomy, MannWhitney and Fisher's exact test were performed, respectively. Kaplan-Meier curves with log-rank analysis were used for comparison of survival. A $p$ value $<0.05$ was considered statistically significant. All analyses were performed using the Predictive Analytics Software 17 (SPSS Inc., Chicago, IL, USA). 


\section{Results}

Patient characteristics

Between May 1989 and June 2010, a total of 160 patients had undergone cystectomy for a urothelial malignancy at Erasmus MC, comprising of 135 (84.4\%) males and $25(15.6 \%)$ females (Table 1). The mean age of the patients at operation was 62.4 years (range 33.282.5 years). The majority of male patients had undergone a radical cystoprostatectomy $(N=128 ; 94.8 \%)$, which was combined by a left $(N=4)$, right $(N=4)$, or bilateral $(N=1)$ nephro-ureterectomy. Four $(3.0 \%)$ male patients had been treated by simple cystectomy, of whom three had undergone a previous radical prostatectomy for prostate adenocarcinoma. Three men $(2.2 \%)$ had been treated by partial cystectomy. A total of 17 (68\%) female patients had undergone a cysto-hysterectomy with adnexa extirpation, combined with a left $(N=1)$ or right $(N=1)$ nephro-ureterectomy. Eight (32\%) females had been treated with simple cystectomy (Table 1).

The clinical indication for cystectomy was UCC with MD invasion ( $\geq \mathrm{cT} 2)$ in 137 patients (85.7\%), therapy-

Table 1 Clinical characteristics of 160 patients operated for urothelial cell carcinoma

\begin{tabular}{ll}
\hline$N=160$ & No. of patients (\%) \\
\hline $\begin{array}{l}\text { Age (median; range) } \\
\text { Sex }\end{array}$ & $62.4(33.2-82.5)$ \\
$\quad$ Male & $135(84)$ \\
Female & $25(16)$ \\
Type of operation & \\
Male & \\
$\quad$ Radical cystoprostatectomy & $128(95)$ \\
$\quad$ With nephro-ureterectomy & 9 \\
$\quad$ Simple cystectomy & $4(3)$ \\
$\quad$ Partical cystectomy & $3(2)$ \\
Female & \\
$\quad$ Cysto-hysterectomy with adnexa & $17(68)$ \\
$\quad$ extirpation & 2 \\
$\quad$ With nephro-ureterectomy & $8(32)$ \\
$\quad$ Simple cystectomy & \\
Indication for cystectomy & $137(86)$ \\
Musculus detrusor invasion & $17(11)$ \\
Therapy-resistant recurrent pTa/pT1 or CIS & $4(2)$ \\
Involvement of the prostatic urethra & $1(0.5)$ \\
by UCC/CIS & $1(0.5)$ \\
Very large superficial high grade UCC & \\
Urothelial CIS combined with sigmoid & \\
adenocarcinoma &
\end{tabular}

$U C C$ urothelial cell carcinoma, $H G$ high grade, $C I S$ carcinoma in situ

resistant recurrence of superficial high grade $\mathrm{pTa} / \mathrm{pT} 1$ UCC and/or CIS ( $N=17 ; 10.6 \%)$, involvement of the prostatic urethra by superficial high grade UCC/CIS $(N=4$; $2.5 \%$ ), a very large superficial UCC (pTa; $N=1 ; 0.6 \%$ ), and urothelial CIS combined with sigmoid adenocarcinoma with potential involvement of the urinary bladder $(N=1$; $0.6 \%$ ). Of the 137 patients who had been operated for MD invasion, 23 (16.8\%) patients had undergone neoadjuvant therapy and $8(5.8 \%)$ had clinical MD invasion without pathological confirmation. For further analysis, we included the remaining $106(77.4 \%)$ patients who had pathologically confirmed MD invasion ( $\geq \mathrm{pT} 2)$ at diagnostic TUR, followed by cystectomy without neo-adjuvant therapy.

\section{Characterization of down-staged UCC}

Of the 106 patients with $\geq$ pT2 UCC at TUR, histologic evaluation of the cystectomy specimen revealed 13 cases (12.3\%) without residual tumor (pT0), 5 (4.7\%) with CIS only, $3(2.8 \%)$ with pTa, $6(5.7 \%)$ with pT1, $33(31.1 \%)$ with pT2, $37(34.9 \%)$ with pT3, and $9(8.5 \%)$ with pT4 disease. Therefore, $27(25.5 \%)$ cases had lower stage UCC at cystectomy, then at initial diagnostic TUR, which showed $\geq \mathrm{pT} 2$ disease (Table 2). No statistically significant difference in age $(p=0.64)$, gender $(p=1.00)$, or number of days between diagnostic TUR and operation $(p=0.96)$ existed

Table 2 Tumor characteristics of 106 patients with $\geq \mathrm{pT} 2$ on diagnostic transurethral resection (TUR), followed by cystectomy for urothelial cell carcinoma without (neo-) adjuvant therapy

CIS carcinoma in situ, PUNLMP papillary urothelial neoplasm of low malignant potential, $L G$ low grade, $H G$ high grade

\begin{tabular}{ll}
\hline$N=106$ & No. of patients (\%) \\
\hline Stage & $13(12)$ \\
pT0 & $5(5)$ \\
pTis & $3(3)$ \\
pTa & $6(6)$ \\
pT1 & $33(31)$ \\
pT2 & $46(43)$ \\
>pT2 & 16 \\
Concomitant & \\
CIS & \\
Grade & \\
WHO 1973 & $13(12)$ \\
No tumor & $0(0)$ \\
G1 & $9(9)$ \\
G2 & $79(79)$ \\
G3 & $13(12)$ \\
WHO 2004 & $0(0)$ \\
No tumor & $0(0)$ \\
PUNLMP & $88(88)$ \\
LG & \\
HG & \\
\hline
\end{tabular}


Table 3 Clinical $(N=106)$ and pathologic $(N=58)$ characteristics of down-staged $(<\mathrm{pT} 2)$ and $\geq \mathrm{pT} 2$ urothelial carcinoma at cystectomy

\begin{tabular}{|c|c|c|c|c|}
\hline No. & Clinico-pathologic variable & Down-staged $<\mathrm{pT} 2$ & $\geq \mathrm{pT} 2$ & $p$ value \\
\hline \multirow[t]{3}{*}{106} & Age (median; range) & $64.9(35.1-73.9)$ & $64.1(33.2-82.5)$ & 0.64 \\
\hline & Male/female (\%) & $23: 4(85 / 15)$ & $68: 11(86 / 14)$ & 1.00 \\
\hline & Time after TUR (days) (median; range) & $57(15-113)$ & $55(12-220)$ & 0.96 \\
\hline \multirow[t]{2}{*}{58} & Number slides (median; range) & $10(3-33)$ & $10(2-23)$ & 0.62 \\
\hline & TUR reaction (\%) & $20 / 27(74)$ & $17 / 31(55)$ & 0.13 \\
\hline
\end{tabular}

Review of pathologic cystectomy characteristics was performed only on the down-staged $(<\mathrm{pT} 2)$ and pT2 urothelial cancers TUR transurethral resection

between down-staged ( $<\mathrm{pT} 2)$ and concordantly staged $(\geq \mathrm{pT} 2)$ cystectomies (Table 3 ).

To investigate whether down-staging was due to difference in sampling, we histologically reviewed all cystectomy slides of $\leq$ pT2 UCC. Stages pT3 and pT4 were not evaluated $(N=46)$. In two cases, slides were not available for review, leaving a total of 58 cases for analysis. The mean number of slides sampled from the urinary bladder was ten in both the down-staged and the concordantly staged pT2 group ( $p=0.62)$. Histological reaction to previous TUR was found in 20/27 (74 \%) cases of the down-staged group as compared to $17 / 31(55 \%)$ cases in the pT2 group ( $p=0.13)$, indicating that under-sampling of the cystectomy specimen was not the cause of tumor down-staging after operation (Table 3).

In order to find histological features at diagnostic TUR that corresponded with down-staging at cystectomy, we reviewed all diagnostic TUR slides of the 58 patients with $\leq$ pT2 UCC. Six cases were not available for review, leaving 52 cases for further analysis. At review, one case showed extensive histiocytic infiltration while no UCC was found in the diagnostic TUR specimen, which was verified with
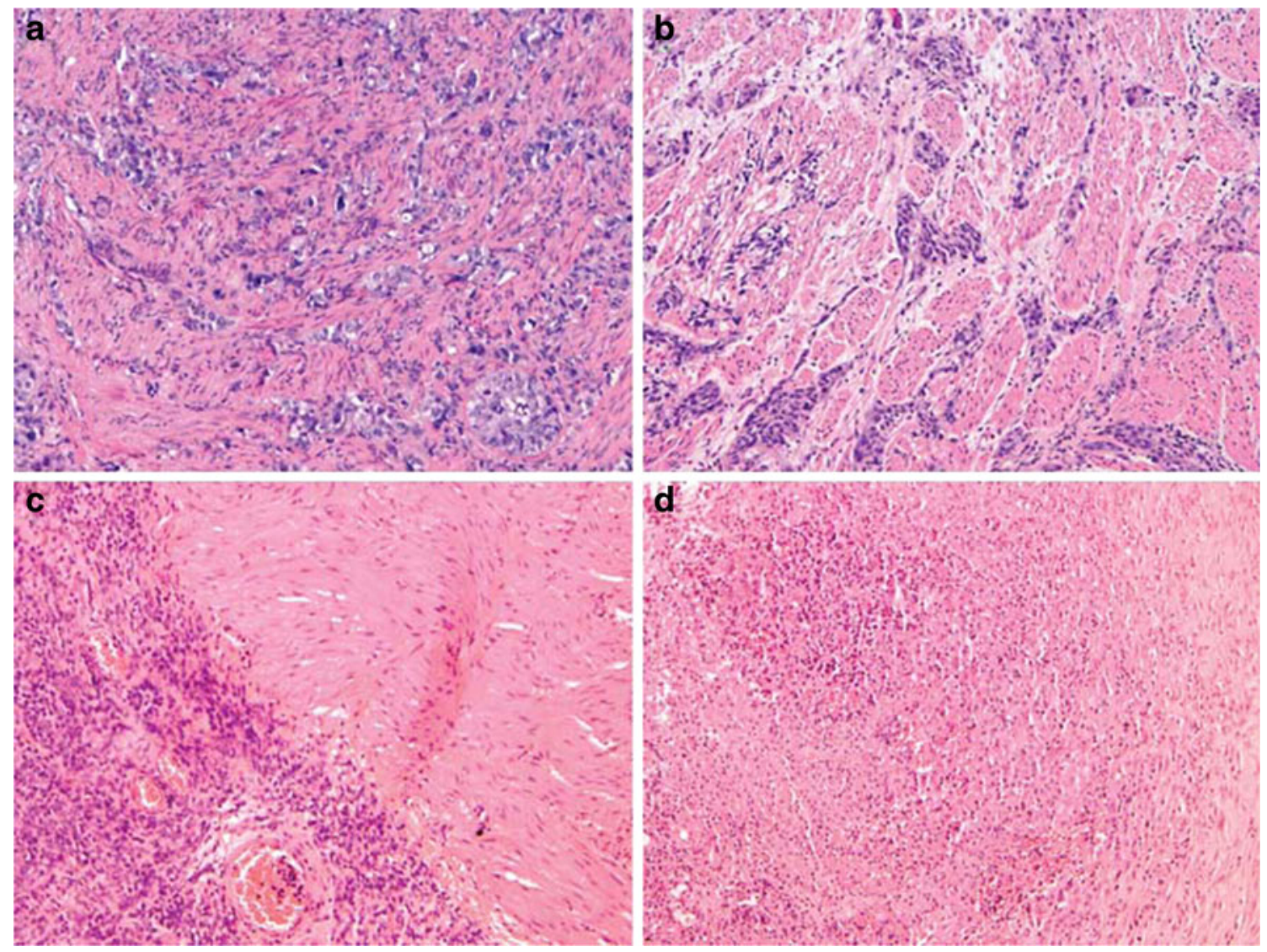

Fig. 1 Assessment of urothelial cell carcinoma invasion in relation to the musculus detrusor. a, b Urothelial carcinoma invading in between musculus detrusor fascicles. $\mathbf{c}$ Urothelial cancer located adjacent to but no in

between musculus detrusor fascicles. $\mathbf{d}$ Histiocytic reaction after previous diagnostic transurethral resection, misinterpreted as urothelial cancer invading the musculus detrusor. HE; original magnifications $\times 100$ 

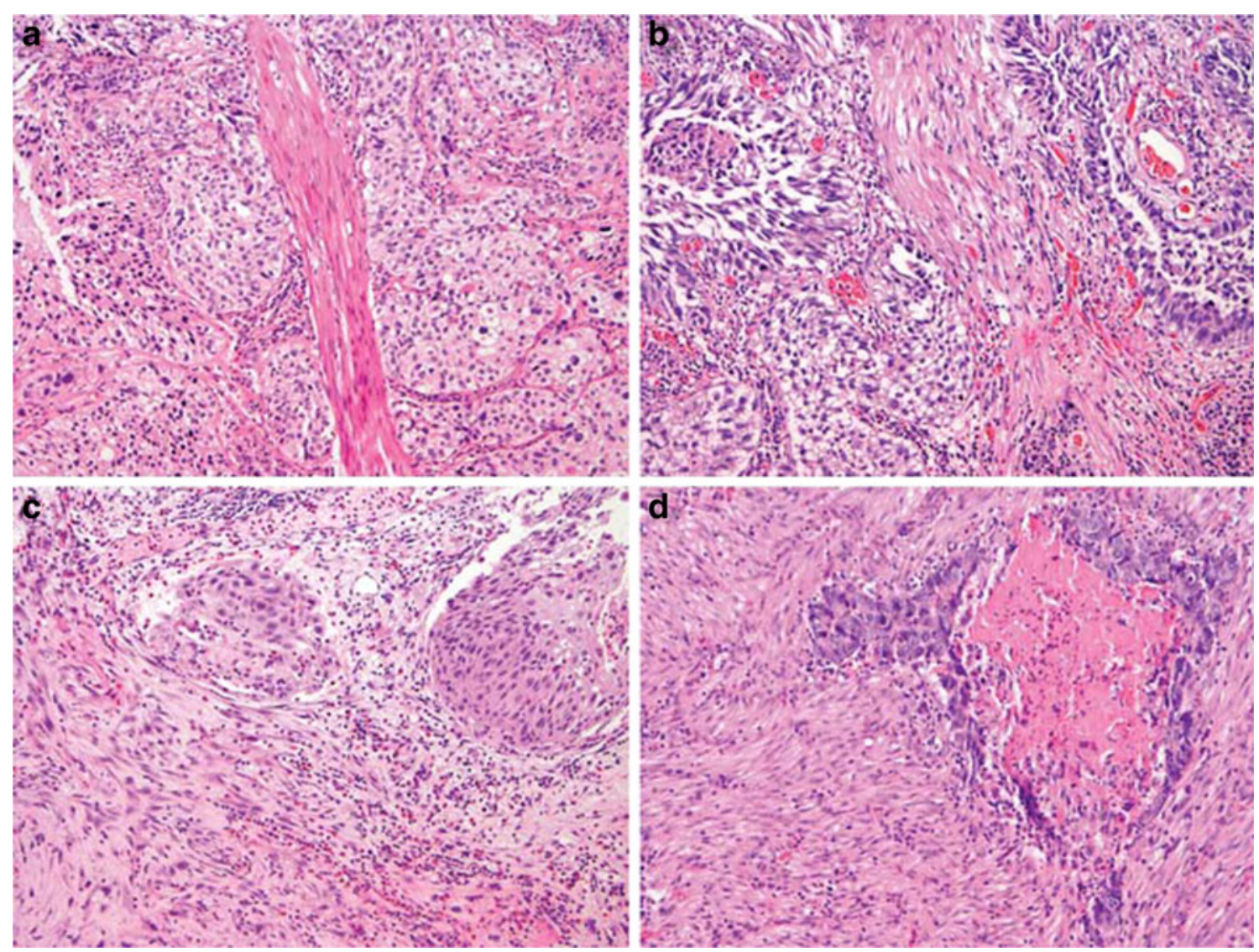

Fig. 2 Pitfalls of urothelial cell carcinoma invasion of musculus detrusor. a, b Muscularis mucosae fascicles mimicking musculus detrusor. c, d Extensive desmoplastic stromal reaction misinterpreted as musculus detrusor invasion. HE; original magnifications $\times 100$


Fig. 3 Survival analysis of down-staged and pT2-staged urothelial cell carcinoma after cystectomy. There was no difference in overall a $(p=0.53)$ or disease-specific $\mathbf{b}(p=0.76)$ survival of patients with $<$ pT2 versus $\mathrm{pT} 2$ urothelial cancer at cystectomy 
negative immunohistochemical Ker7 staining (Fig. 1). This case was incorrectly diagnosed as UCC at the time of diagnosis; subsequent cystoprostatectomy indeed did not reveal UCC (pT0). In the remaining 51 cases, grade according to WHO $1973(p=0.28)$ or $2004(p=0.26)$, and concomitant CIS $(p=0.55)$ were not associated with down-staging at cystectomy. Of the tumors, 40/51 $(78.4 \%)$ demonstrated invasion into the MD, 4 (7.8\%) showed tumor adjacent to the MD but not in between the muscle fascicles, and $7(13.5 \%)$ did not reveal invasion up to MD bundles convincingly (Fig. 2). Of these seven cases, four $(7.7 \%)$ showed invasion in between small smooth muscle fascicles, which were not characteristic for $\mathrm{MD}$, but could represent $\mathrm{MM}$ and three $(5.8 \%)$ demonstrated extensive desmoplastic stromal reaction without tumor infiltration into the MD. The desmoplastic stromal reaction was probably interpreted as MD invasion at initial diagnosis (Fig. 3). In four cases, Desmin immunohistochemistry expression was used to facilitate identification of smooth muscle fascicles at review, in case of cauterization or differentiation with desmoplastic stromal reaction. There was no significant relation of the type of tumor invasion in the TUR specimen and the subsequent findings at cystectomy (Table 4).

\section{Survival analysis}

The mean follow-up of the pT2 group and the downstaged group was 61.6 and 70.9 months, respectively. To investigate whether patients who were down-staged at cystectomy had a favorable outcome versus those with stage pT2 at cystectomy, survival analysis was performed. We excluded 9/60 (15\%) patients with positive regional lymph nodes from the analysis, i.e., $4 / 27(14.8 \%)$ in the down-staged group and 5/33 $(15.2 \%)$ in the pT2 group. There was no significant difference in overall $(p=0.53)$ and disease-specific $(p=$ $0.76)$ survival between the adequately staged and downstaged group (Fig. 3).

\section{Discussion}

In a significant number of cases, urologists and pathologists are confronted with UCC down-staged $(<\mathrm{pT} 2)$ at cystectomy, although operation was prompted by an MD invasive tumor at diagnostic TUR. Down-staging at cystectomy might have several causes, such as successful neo-adjuvant therapy, complete UCC resection at TUR, or false-positive diagnosis at TUR. In the present study, we investigated the incidence and the possible origin of down-staging in a welldefined cohort of 106 cystectomy patients, of whom operation was prompted by pathologic MD invasion at diagnostic TUR and who had not undergone neo-adjuvant therapy.

We found down-staging at cystectomy in $26 \%$ of the study population. In $12 \%$, no residual UCC (pT0) was identified, which is in line with numbers (6-20\%) reported by others [6-9]. Age, gender, and interval between TUR and operation were not statistically different between concordantly staged pT2 and the down-staged group. Since the number of slides sampled at grossing and frequency of identifying a TUR scar at microscopic evaluation of cystectomy specimens was similar in both groups, down-staging was not due to sampling error.

At review of the diagnostic TUR specimens, in 12 out of $52(23.1 \%)$ cases, UCC infiltration into the MD could not be confirmed. Discrepancy with the original TUR staging was caused by false interpretation of (a) histiocytic reaction as invasive UCC (1.9\%), (b) invasion of the MM as MD invasion $(7.7 \%)$, (c) extensive desmoplastic stromal reaction as MD (5.8\%), and (d) UCC impinging upon the MD as invasion into the MD (7.7\%). As far as we know, the latter subtle distinction has not been subject of study yet. If UCC adjacent to MD would be classified as pT2 disease, as is often done in pathologic practice, the prevalence of downstaging UCC at review of TUR specimens was $15.4 \%(8 / 52)$. Interestingly, this percentage of staging discordance is in line with the percentage of original pT1 UCC up-staged to pT2 disease at review, indicating that inter-observer variability is also existing in assessing MD invasion [11]. Distinguishing between MM and MD invasion at diagnostic TUR is a wellknown challenge in surgical pathology, which is hampered
Table 4 Pathologic characteristics of urothelial cell carcinoma at diagnostic transurethral resections (TUR) in the down-staged $(N=21)$ and adequately pT2staged group $(N=30)$

$L G$ low grade, $H G$ high grade, CIS carcinoma in situ, $M M$ muscularis mucosae, $M D$ musculus detrusor

\begin{tabular}{llll}
\hline$N=51$ & $\begin{array}{l}\text { Down-staged } \\
N(\%)\end{array}$ & $\begin{array}{l}\text { Adequate pT2 } \\
N(\%)\end{array}$ & $p$ value \\
\hline G2/G3 & $2 / 19(10 / 90)$ & $7 / 23(23 / 77)$ & 0.28 \\
LG/HG & $0 / 21(0 / 100)$ & $3 / 27(10 / 90)$ & 0.26 \\
Concomitant CIS & $8(38)$ & $9(30)$ & 0.55 \\
Desmoplastic stromal reaction & $0(0)$ & $3(100)$ & N/A \\
MM invasion & $2(50)$ & $2(50)$ & 1.00 \\
Adjacent to MD & $2(50)$ & $2(50)$ & 1.00 \\
In between MD fascicles & $17(43)$ & $23(57)$ & 1.00 \\
\hline
\end{tabular}


by problems in tissue orientation and potential MM hyperplasia. In addition, MD-infiltrating tumors may partially destruct muscle fibers, leaving thin residual MD bundles resembling MM [12]. Despite these difficulties, accurate recognition of MD invasion is crucial for patient treatment according to the current guidelines. Immunohistochemical expression of the cytoskeletal protein Smoothelin is reported to be stronger in MD than in MM [13-15]. Therefore, additional immunohistochemistry using Smoothelin can be useful in differentiating both muscle layers. While variable Smoothelin expression patterns can be appreciated when both layers are present in the same specimen, its value in classifying a limited amount of smooth muscle fibers in small specimens remains to be established. Therefore, we decided not to include this marker in the current study. Finally, extensive desmoplastic myofibroblastic reaction evoked by UCC invasion might histologically mimic MD [16]. In this case, immunohistochemical detection of Desmin is helpful to distinguish myofibroblasts from MD smooth muscle cells [17].

No statistically significant difference in overall and disease-specific survival was found between the downstaged and concordantly staged pT2 group. Interestingly, the percentage of patients with positive lymph nodes was similar between the two groups as well. Regarding the patients with pT0 disease, survival after cystectomy was not different from the patients with higher tumor stages. This is in accordance with the findings of Thrasher et al. [18]. This implicates that MD invasive UCC at diagnostic TUR with down-staging at cystectomy should still be regarded as pT2 disease. The clinical significance of pT0 disease at cystectomy is, however, still under debate. Volkmer et al. excluded patients with neo-adjuvant therapy and found a significantly better disease-specific survival in patients with $\mathrm{pT} 0$ disease at cystectomy versus patients with maximal pT2a disease at cystectomy [19]. Likewise, Tilki et al. investigated a group of 4,430 patients treated with radical cystectomy and found significantly better clinical outcomes in patients with pT0 disease than those with clinical and pathological stage T2 [20]. These results are conflicting with the findings in our study, which could be explained by the fact that in our study a relatively small number of patients were included.

This relatively low number of patients is a limitation of this study and was due to our strict selection criteria, i.e., exclusion of non-urothelial cancer such as squamous carcinoma and adenocarcinoma, presence of neo-adjuvant therapy, availability of slides for review, and omitting high-stage disease (pT3/4) from analysis. Nevertheless, to the best of our knowledge, this is the first study specifically investigating the nature of down-staging in UCC, in which we retrospectively demonstrate $15 \%$ inter-observer variability in the diagnosis of clinically decisive MD invasion. For practical purposes, important lessons can be drawn from this study. First, UCC invasion directly adjacent to MD bundles can be classified as pT2 disease at TUR since down-staging in this group did not occur more frequently than in UCC with invasion into the MD fascicles. Secondly, the presence of pT2 disease at cystectomy in patients with MM invasion or extensive desmoplastic stromal reaction yet no MD invasion at TUR as well as the significant inter-observer variability of T1-2 staging raises the question whether the current clinicopathologic guidelines for treatment of invasive UCC can be optimized. Herr performed a re-staging TUR in patients with histologically proven pT2 disease [21]. If re-staging revealed pT0 or pT1 disease, patients were offered immediate cystectomy or careful follow-up. Interestingly, the 10-year disease-specific survival was similar in both groups with significantly better survival in patients with $\mathrm{pT} 0$ as compared to pT1 tumor at re-staging. Since cystectomy is a surgical procedure with 90 -day complication rates as high as $46 \%$ and peri-operative mortality ranging from 1 to $4 \%[3,22]$, further studies on the use of re-TUR and imaging modalities for the treatment and follow-up as alternative for radical operation in a subgroup of UCC patients are mandatory.

In conclusion, we have shown that $26 \%$ of patients with initial pT2 stage at diagnostic TUR were down-staged at subsequent cystectomy. No clinical or pathologic parameters could be identified that were associated with downstaging $(<\mathrm{pT} 2)$ of UCC at cystectomy. Review of diagnostic TUR specimens revealed inter-observer variability, with pT 2 to $<$ pT 2 in $15 \%$ of the cases. No difference in survival was found in patients with down-staged versus pT2 UCC at cystectomy.

Conflict of interest The authors declare that they have no conflict of interest.

Open Access This article is distributed under the terms of the Creative Commons Attribution License which permits any use, distribution, and reproduction in any medium, provided the original author(s) and the source are credited.

\section{References}

1. Jemal A, Siegel R, Ward E, Hao Y, Xu J, Murray T, Thun MJ (2008) Cancer statistics, 2008. CA Cancer J Clin 58:71-96

2. Colombel M, Akaza H, Bohle A, Palou J, Buckley R, Lamm D, Brausi M, Witjes JA, Persad R (2008) Epidemiology, staging, grading, and risk stratification of bladder cancer. Eur Urol Suppl 7:618-626

3. Morgan TM, Keegan KA, Clark PE (2011) Bladder cancer. Curr Opin Oncol 23:275-282

4. Ghoneim MA, Abdel-Latif M, el-Mekresh M, Abol-Enein H, Mosbah A, Ashamallah A, el-Baz MA (2008) Radical cystectomy for carcinoma of the bladder: 2,720 consecutive cases 5 years later. J Urol 180:121-127 
5. Shariat SF, Karakiewicz PI, Palapattu GS, Lotan Y, Rogers CG, Amiel GE, Vazina A, Gupta A, Bastian PJ, Sagalowsky AI, Schoenberg MP, Lerner SP (2006) Outcomes of radical cystectomy for transitional cell carcinoma of the bladder: a contemporary series from the Bladder Cancer Research Consortium. J Urol $176: 2414-2422$

6. Dalbagni G, Genega E, Hashibe M, Zhang ZF, Russo P, Herr H, Reuter V (2001) Cystectomy for bladder cancer: a contemporary series. J Urol 165:1111-1116

7. Lee SE, Jeong IG, Ku JH, Kwak C, Lee E, Jeong JS (2004) Impact of transurethral resection of bladder tumor: analysis of cystectomy specimens to evaluate for residual tumor. Urology 63:873-877

8. Amling CL, Thrasher JB, Frazier HA, Dodge RK, Robertson JE, Paulson DF (1994) Radical cystectomy for stages Ta, Tis and T1 transitional cell carcinoma of the bladder. J Urol 151:31-35

9. Stein JP, Lieskovsky G, Cote R, Groshen S, Feng AC, Boyd S, Skinner E, Bochner B, Thangathurai D, Mikhail M, Raghavan D, Skinner DG (2001) Radical cystectomy in the treatment of invasive bladder cancer: long-term results in 1,054 patients. J Clin Oncol 19:666-675

10. Svatek RS, Shariat SF, Novara G, Skinner EC, Fradet Y, Bastian PJ, Kamat AM, Kassouf W, Karakiewicz PI, Fritsche HM, Izawa JI, Tilki D, Ficarra V, Volkmer BG, Isbarn H, Dinney CP (2011) Discrepancy between clinical and pathological stage: external validation of the impact on prognosis in an international radical cystectomy cohort. BJU Int 107:898-904

11. Bol MG, Baak JP, Buhr-Wildhagen S, Kruse AJ, Kjellevold KH, Janssen EA, Mestad O, Ogreid P (2003) Reproducibility and prognostic variability of grade and lamina propria invasion in stages Ta, T1 urothelial carcinoma of the bladder. J Urol 169:1291-1294

12. Ro JY, Ayala AG, el-Naggar A (1987) Muscularis mucosa of urinary bladder. Importance for staging and treatment. Am J Surg Pathol 11:668-673

13. Miyamoto H, Sharma RB, Illei PB, Epstein JI (2010) Pitfalls in the use of smoothelin to identify muscularis propria invasion by urothelial carcinoma. Am J Surg Pathol 34:418-422
14. Paner GP, Brown JG, Lapetino S, Nese N, Gupta R, Shen SS, Hansel DE, Amin MB (2010) Diagnostic use of antibody to smoothelin in the recognition of muscularis propria in transurethral resection of urinary bladder tumor (TURBT) specimens. Am J Surg Pathol 34:792-799

15. Paner GP, Shen SS, Lapetino S, Venkataraman G, Barkan GA, Quek ML, Ro JY, Amin MB (2009) Diagnostic utility of antibody to smoothelin in the distinction of muscularis propria from muscularis mucosae of the urinary bladder: a potential ancillary tool in the pathologic staging of invasive urothelial carcinoma. Am J Surg Pathol 33:91-98

16. Cheng L, Montironi R, Davidson DD, Lopez-Beltran A (2009) Staging and reporting of urothelial carcinoma of the urinary bladder. Mod Pathol 22:S70-S95

17. Council L, Hameed O (2009) Differential expression of immunohistochemical markers in bladder smooth muscle and myofibroblasts, and the potential utility of desmin, smoothelin, and vimentin in staging of bladder carcinoma. Mod Pathol 22:639-650

18. Thrasher JB, Frazier HA, Robertson JE, Paulson DF (1994) Does of stage pT0 cystectomy specimen confer a survival advantage in patients with minimally invasive bladder cancer? J Urol 152:393-396

19. Volkmer BG, Kuefer R, Bartsch G Jr, Straub M, de Petriconi R, Gschwend JE, Hautmann RE (2005) Effect of a pT0 cystectomy specimen without neoadjuvant therapy on survival. Cancer 104:2384-2391

20. Tilki D, Svatek RS, Novara G, Seitz M, Godoy G, Karakiewicz PI, Kassouf W, Fradet Y, Fritsche HM, Sonpavde G, Izawa JI, Ficarra V, Lerner SP, Schoenberg M, Stief CG, Dinney CP, Skinner E, Lotan Y, Sagalowsky AI, Reich O, Shariat SF (2010) Stage pT0 at radical cystectomy confers improved survival: an international study of 4,430 patients. J Urol 184:888-894

21. Herr HW (2001) Transurethral resection of muscle-invasive bladder cancer: 10-year outcome. J Clin Oncol 19:89-93

22. Morgan TM, Clark PE (2010) Bladder cancer. Curr Opin Oncol 22:242-249 